\title{
African crop yield reductions due to increasingly unbalanced Nitrogen and Phosphorus consumption
}

Marijn van der Velde (1,2), Christian Folberth (2,3), Juraj Balkovič (2,4), Philippe Ciais (5), Steffen Fritz (2), Ivan A. Janssens (6), Michael Obersteiner (2), Linda See (2), Rastislav Skalský (2), Wei Xiong (2), and Josep Peñuealas (7)

(1) European Commission DG-JRC IES/MARS, Institute for the Environment, Ispra, Italy (marijnvandervelde@ gmail.com), (2) International Institute of Applied Systems Analysis (IIASA), Ecosystem Services and Management Program, Schlossplatz 1, Laxenburg, A-2361, Austria, (3) Systems Analysis Integrated Assessment and Modelling, Swiss Federal Institute of Aquatic Science and Technology (EAWAG), Ueberlandstr. 133, Deubendorf CH-8600, Switzerland, (4) Department of Soil Science, Faculty of Natural Sciences, Comenius University in Bratislava, Bratislava 842 15, Slovak Republic, (5) Laboratoire des Sciences du Climat et de l'Environnement, IPSL, Gif-sur-Yvette, France, (6) Department of Biology, University of Antwerp, Universiteitsplein 1, Wilrijk B-2610, Belgium, (7) Global Ecology Unit CREAF-CEAB-UAB, CSIC, Cerdanyola del Valles, Catalonia 08193 Spain, 7CREAF, Cerdanyola del Valles, Catalonia 08193, Spain

The impact of soil nutrient depletion on crop production has been known for decades, but robust assessments of the impact of increasingly unbalanced nitrogen $(\mathrm{N})$ and phosphorus $(\mathrm{P})$ application rates on crop production are lacking. Here, we use crop response functions based on 741 FAO maize crop trials and EPIC crop modeling across Africa to examine maize yield deficits resulting from unbalanced N:P applications under low, medium, and high input scenarios, for past (1975), current, and future N:P mass ratios of respectively, 1:0.29, 1:0.15, and 1:0.05. At low $\mathrm{N}$ inputs (10 kg/ha), current yield deficits amount to $10 \%$ but will increase up to $27 \%$ under the assumed future N:P ratio, while at medium $\mathrm{N}$ inputs ( $50 \mathrm{~kg} \mathrm{~N} / \mathrm{ha}$ ), future yield losses could amount to over $40 \%$. The EPIC crop model was then used to simulate maize yields across Africa. The model results showed relative median future yield reductions at low $\mathrm{N}$ inputs of $40 \%$, and $50 \%$ at medium and high inputs, albeit with large spatial variability. Dominant low-quality soils such as Ferralsols, which are strongly adsorbing $\mathrm{P}$, and Arenosols with a low nutrient retention capacity, are associated with a strong yield decline, although Arenosols show very variable crop yield losses at low inputs. Optimal N:P ratios, i.e. those where the lowest amount of applied P produces the highest yield (given $\mathrm{N}$ input) where calculated with EPIC to be as low as 1:0.5. Finally, we estimated the additional P required given current $\mathrm{N}$ inputs, and given $\mathrm{N}$ inputs that would allow Africa to close yield gaps (ca. 70\%). At current $\mathrm{N}$ inputs, $\mathrm{P}$ consumption would have to increase 2.3 -fold to be optimal, and to increase 11.7-fold to close yield gaps. The $\mathrm{P}$ demand to overcome these yield deficits would provide a significant additional pressure on current global extraction of $\mathrm{P}$ resources. 